Nature Reviews Molecular Cell Biology | Published online 19 Dec 2016; doi:10.1038/nrm.2016.165

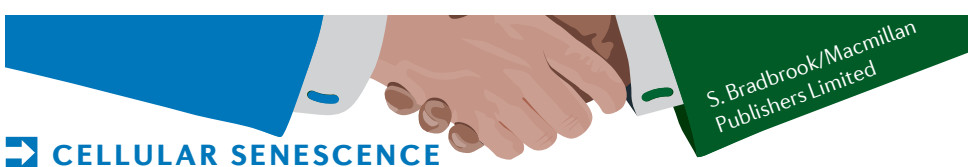

\title{
Senescence and reprogramming go hand-in-hand
}

The mechanisms underlying transcription factor-mediated reprogramming of somatic differentiated cells to a pluripotent state have been extensively studied in vitro, but the mechanisms driving and regulating reprogramming in vivo are still largely unknown. Serrano and colleagues now report that, in mice, the expression of the four transcription factors OCT4, SOX2, KLF4 and MYC (together referred to as 'OSKM'), which induces pluripotency, also induces cellular senescence, and that senescent cells produce signals that facilitate the reprogramming of neighbouring cells.

First, the authors tested whether reprogramming in vivo is facilitated by the removal of the tumour suppressors p53, INK4A and ARF, as these proteins are known to inhibit reprogramming in vitro. They generated transgenic mice that express OSKM in an inducible manner and that are deficient for Trp53 (which encodes p53) or Cdkn2a (the locus that encodes INK4A and ARF). Upon OSKM expression, Trp53deficient mice displayed a higher rate of teratoma formation (an assay for pluripotency) and induction of the expression of pluripotency marker Nanog as expected. But, surprisingly, the opposite was observed in Cdkn2adeficient mice, which were highly resistant to teratoma formation and devoid of Nanog-positive cells, indicating that INK4A and ARF promote reprogramming in vivo.

As it is well known that the Cdkn2a locus is involved in cellular senescence, the authors investigated whether senescence and reprogramming might be linked. Interestingly, Nanog-positive cells were often found in close proximity to clusters of cells expressing markers of senescence (such as senescence-associated $\beta$-galactosidase and the cell cycle inhibitor p21). Moreover, whereas in Trp53-deficient mice there was a strong senescence and inflammatory response following OSKM induction, this response was minimal in $C d k n 2 a$ deficient mice. Thus, the authors suggest that OSKM induce both cellular reprogramming and damage-induced cellular senescence, but the latter is impaired in Cdkn2a-deficient mice.

Next, the authors went on to test whether senescent cells and their secretory activity (known as senescence-associated secretory phenotype (SASP)) induce reprogramming. Treatment of mice with small molecules that reduce the number of senescent cells led to a decrease in the number of Nanog-positive cells and thus reprogramming. Moreover, treatment with antibodies against interleukin-6 (IL-6), a SASP factor that was upregulated upon OSKM induction (and is a known inducer of reprogramming in vitro), reduced reprogramming efficiency, senescence and SASP. These results indicate that IL- 6 is a crucial cytokine that links senescence and reprogramming in vivo.

Interestingly, the link between tissue damage, senescence and reprogramming was also seen in the context of increased senescence, such as tissue injury and ageing. Expression of OSKM in injured tissues, and in progeric mice or naturally old mice, induced higher levels of reprogramming compared with normal mice.

Together, this work shows that ectopic expression of OSKM in vivo induces both cellular reprogramming and senescence and that the latter facilitates the process of reprogramming to pluripotency through the secretion of key cytokines.

Kim Baumann

ORIGINAL ARTICLE Mosteiro, L. et al. Tissue damage and senescence provide critical signals for cellular reprogramming in vivo. Science http:// dx.doi.org/10.1126/science.aaf4445 (2016) FURTHER READING Takahashi, K. \& Yamanaka, S. A decade of transcription factor-mediated reprogramming to pluripotency. Nat. Rev. Mol. Cell Biol. 17, 183-193 (2016) | Muñoz-Espín, D. 8 Serrano, M. Cellular senescence: from physiology to pathology. Nat. Rev. Mol. Cell Biol. 15, 482-496 (2014) 